\title{
A DEFORMATION THEOREM FOR THE KOBAYASHI METRICI
}

\author{
M. KALKA
}

\begin{abstract}
Let $M_{0}$ be a compact hyperbolic complex manifold. It is shown that the infinitesimal Kobayashi metric is upper semicontinuous in a $C^{\infty}$ deformation parameter $t \in U \subseteq R^{k}$. This is accomplished by proving deformation theorems for holomorphic maps.
\end{abstract}

Introduction and statement of results. In this paper we examine the behavior of compact hyperbolic complex manifolds under small perturbations of the complex structure. In particular we prove a semicontinuity theorem for Royden's infinitesmal version of the Kobayashi metric. M. Wright [9] had obtained these results a year prior to our work. His paper was not submitted for publication until our work was completed, and our work is independent of his. Brody [1] has recently shown that the property of being compact hyperbolic is preserved under small pertubations of the complex structurethereby answering, in the affirmative, a question of Griffiths [4].

We will use the following notation throughout: $D_{r}=\{z \in \mathbf{C}|| z \mid<r\}$, if we write $D$ we mean $D_{1} . D^{k}=\left\{z \in \mathbf{C}^{k}|| z^{i} \mid<1\right\}$. The holomorphic tangent bundle of a complex manifold $X$ will be denoted by $\mathscr{T} X$ and the holomorphic sphere bundle to a complex manifold $X$ (once a hermitian metric is fixed) will be denoted by $\delta X$. If the complex structure of a manifold $M$, depends on a parameter $t$, varying over some open set $U \subseteq \mathbf{R}^{k}$, then $M_{t}$ will denote the $C^{\infty}$ manifold in the $t$-complex struct:ure.

If $M$ is a complex manifold then $d_{M}(p, q)$ is the Kobayashi pseudo-distance between $p$ and $q$. If $(x, \xi) \in \Im M, F_{M}(x, \xi)$ will denote Royden's infinitesmal version of the Kobayashi metric.

From the definition of the Kobayashi metric it is clear that what is needed is a result on deformation of holomorphic maps of the unit disc in $\mathbf{C}$ into the manifold in question. This is accomplished using Kohn's solutions of the $\bar{\partial}$ Neumann problem [3] and Royden's extension lemma for regular holomorphic maps [7].

Received by the editors October 9, 1975.

AMS (MOS) subject classifications (1970). Primary 32H20, 32G05.

Key words and phrases. Deformation of complex structure, hyperbolic manifold, infinitesimal Kobayashi metric.

1 This paper contains some of the results of the author's doctoral dissertation at Courant Institute under Louis Nirenberg to whom the author is grateful.

O American Mathematical Society 1976 
Theorem 1. Suppose $M_{0}$ is a complex manifold and $f_{0}: D \rightarrow M_{0}$ is a holomorphic map. Suppose $\left\{M_{t}\right\}_{t \in U}$ is a deformation of the complex structure on $M_{0}$. Then given $r<1 \exists U^{\prime} \subset U$ and maps $f_{t}: D_{r} \rightarrow M_{t}$ so that

(1) $f_{t}$ is holomorphic,

(2) $\left.f_{t}\right|_{t=0}=\left.f_{0}\right|_{D_{r}}$,

(3) $f_{t}$ depends smoothly on $t$,

(4) $f_{t}(0)=f_{0}(0)$,

(5) $d f_{t} /\left.d z\right|_{z=0}=P_{t}\left(d f_{0} /\left.d z\right|_{z=0}\right)$. $P_{t}(v)$ is the projection of $v$ on $\sigma M_{t}$.

Here we have made no assumptions on $M_{0}$ other than it being a complex manifold. If we assume $M_{0}$ is compact hyperbolic then we can get a stronger theorem, to wit:

THEOREM 2. If, in Theorem 1, we require that $M_{0}$ be compact hyperbolic, then we get that $\exists U^{\prime} \subset U$ such that every $f: D \rightarrow M_{0}$ can be deformed to $f_{t}: D_{r}$ $\rightarrow M_{t}$. Here $U^{\prime}$ is independent of $f$ and depends only on $r$ and the deformation.

We use Theorem 2 to prove our main result on Royden's metric:

THEOREM 3. Suppose $M_{0}$ is a compact hyperbolic complex manifold Let $\left\{M_{t}\right\}_{t \in U}$ be a deformation of the complex structure on $M_{0}$. Let $\varepsilon>0$ be arbitrary. Then $\exists U_{\varepsilon}^{\prime} \subset U$ so that if $t \in U_{\varepsilon}^{\prime}$ we have

$$
F_{M_{t}}\left(x, P_{t}(\xi)\right) \leqslant F_{M_{0}}(x, \xi)+\varepsilon, \quad \text { for }(x, \xi) \in \delta M_{0}
$$

in some fixed Hermitian metric.

1. Preliminaries. We recall the definition of Royden's infinitesmal version of the Kobayashi metric. Let $(x, \xi) \in \mathcal{T} M_{0}$; then

$$
F_{M_{0}}(x, \xi)=\inf _{R}\left\{\frac{1}{R}, \exists f: D_{R} \rightarrow M_{0}, f(0)=x, f^{\prime}(0)=\xi, f \text { holomorphic }\right\} .
$$

We remark (i) Royden [6] has proven that $F_{M_{0}}$ is the infinitesimal version of the Kobayashi metric in the sense that

$$
d_{M_{0}}(p, q)=\inf _{\gamma \in \Gamma(p, q)} \int_{\gamma} F_{M}\left(x(t), \dot{x}(t)_{(1,0)}\right) d t
$$

and $\Gamma(p, q)$ is the set of all piecewise smooth curves $\gamma:[0,1] \rightarrow M, \gamma(0)^{\circ}=p$, $\gamma(1)=q[6]$.

(ii) $F_{M_{0}}$ is homogeneous of degree 1 in the $\xi$-variable so that $F_{M}$ can be thought of as defined on $\delta M_{0}$.

We will assume that $M_{0}$ is compact hyperbolic and list two relevant properties that hyperbolicity implies. In both cases the proofs are straightforward.

(a) Let $H\left(D, M_{0}\right)$ denote the space of holomorphic mappings of $D$ into $M_{0}$ equipped with the compact-open topology. Then $H\left(D, M_{0}\right)$ is compact.

(b) Let $(x, \xi) \in \mathcal{T} M_{0}$; then 


$$
\exists f_{(x, \xi)}: D_{F_{M_{0}}(x, \xi)^{-1}} \rightarrow M_{0} \quad \text { with } f(0)=x, f^{\prime}(0)=\xi .
$$

(b) follows easily from (a), and (a) follows from the distance decreasing property of the Kobayashi metric and the Arzelà-Ascoli Theorem.

We will be interested in a deformation of the complex structure on $M_{0}$. We will deal locally in the deformation space, so we will assume, using the Newlander-Nirenberg Theorem, that the deformation is given by a holomorphic vector field valued $(0,1)$ form on the original manifold satisfying an integrability condition. More precisely suppose we are given a family of operators $\bar{\partial}_{t}$ on $M$ considered as a $C^{\infty}$ manifold $\bar{\partial}_{t}: C^{\infty}(M) \rightarrow \Lambda^{1}(M)$ with the following properties:

1. In local holomorphic coordinates $z=\left(z^{1}, \ldots, z^{n}\right)$ on $M_{0}$

$$
\bar{\partial}_{t}=\sum_{i}\left(\frac{\partial}{\partial \bar{z}^{i}}+\sum_{k} \varphi_{i}^{k}(t) \frac{\partial}{\partial z^{k}}\right) d \bar{z}^{i} ;
$$

2. $\varphi_{i}^{k}(0) \equiv 0$;

3. $\varphi_{i}^{k}$ is $C^{\infty}$ in both the $t$ and $z$ parameter;

4. $\bar{\partial}_{t}$ extends naturally to an operator $\bar{\partial}_{t}: \Lambda^{k}(M) \rightarrow \Lambda^{k+1}(M)$.

The integrability condition is then that $\left\{\bar{\partial}_{t}\right\}$ should define a complex i.e. $\bar{\partial}_{t}^{2}=0$.

The Cauchy-Riemann operator for the deformed structure $M_{t}$ is then given by $\bar{\partial}_{t}$. It is clear that $\bar{\partial}_{t}$ acts on sections of vector bundles $E_{t}$ which are holomorphic over $M_{t}$, since $\bar{\partial}_{t}$ annihilates the transition matrices.

We will use the following proposition which tells us that holomorphic functions can be chosen to depend smoothly on the deformation parameter, if the original manifold is a strongly pseudo-convex domain with $C^{\infty}$ boundary in a complex manifold. $\Theta(\Omega)$ will denote the space of holomorphic functions on a complex manifold $\Omega$.

Proposition 1.1. Let $\Omega_{0}$ be a strongly pseudo-convex domain with smooth boundary. Let $\zeta_{0} \in \mathcal{O}\left(\Omega_{0}\right) \cap C^{\infty}\left(\bar{\Omega}_{0}\right)$. Then if $\Omega_{t}$ is a deformation of the complex structure on $\Omega_{0}$ smooth on $\partial \Omega_{0}$, we can find $\zeta_{t}$ for $t$ sufficiently small satisfying

1. $\zeta_{t} \in\left(\Omega_{t}\right) \cap C^{\infty}\left(\bar{\Omega}_{t}\right)$,

2. $\zeta_{0}=\left.\zeta_{t}\right|_{t=0}$,

3. $\left\|\zeta_{t}-\zeta_{0}\right\|_{s} \leqslant C\left(s, \zeta_{0}\right)\|\varphi(t)\|_{s}$, where \|\|$_{s}$ is the Sobolev norm in some Hermitian metric.

Proof. We are looking for a solution of $\bar{\partial}_{t} \zeta_{t}=0$. Let $\mu_{t}=\bar{\partial}_{t} \zeta_{0}$. Now since $\Omega_{0}$ is strongly pseudo-convex so is $\Omega_{t}$ for $t$ small. We now use Kohn's solution of the $\bar{\partial}$-Neumann problem to solve $\bar{\partial}_{t} u_{t}=\mu_{t}$ to get a solution $u_{t}$ such that $\|u\|_{s} \leqslant C\|\mu(t)\|_{s}$.

Now consider

$$
\zeta_{t}=\zeta_{0}-u_{t}, \quad \bar{\partial}_{t} \zeta_{t}=\bar{\partial}_{t}\left(\zeta_{0}-u_{t}\right)=0
$$

and 


$$
\left\|\zeta_{t}-\zeta_{0}\right\|_{s}=\left\|u_{t}\right\|_{s} \leqslant C_{1}\|\mu(t)\|_{s} \leqslant C_{2}\left\|\zeta_{0}\right\|_{s+1}\|\varphi(t)\|_{s} .
$$

Remarks. 1. In Proposition $1.1 \zeta_{0}$ need not be a function but can be a holomorphic vector field. What one does in this case is first project $\zeta_{0}$ to the holomorphic tangent bundle of $\Omega_{t}$, and then proceed as in the proof above.

2. If $\Omega$ is biholomorphically equivalent to a domain in $\mathbf{C}^{n}$, we can take the vector field $\zeta_{t}$ at a given point, 0 , to be $p_{t}\left(\zeta_{0}\right)(0)$ the projection of $\zeta_{0}$ on the holomorphic tangent bundle to $\Omega_{t}$ by simply subtracting from $u_{t}$ the constant solution $u_{t}(0)$.

2. Deformations of hyperbolic structure. In this section we will prove the theorems stated in the introduction. Essential to our treatment will be the following theorem of Royden.

Proposition 2.1 [7]. Let $f$ be a holomorphic embedding of $D$ into a complex manifold $M$. Then given $r<1$ there is a holomorphic embedding $F: D_{r} \times D^{n-1}$ $\rightarrow M$ such that

$$
F\left|D_{D_{r} \times\{0\}}=f\right|_{D_{r}} .
$$

REMARK. Royden's theorem is actually for embeddings of the unit polydisk $D^{k}$ into $M$, and has been extended by C. Seabury [8] to embeddings with trivial normal bundle of a Stein manifold into a complex manifold. We, however, only need the case of $D$.

Proof of Theorem 1 . We consider the case of an embedding. Let $F_{0}$ be the extension of $f_{0}$ to $D_{r^{\prime}} \times D^{n-1}, r^{\prime}>r$, given by Royden's theorem. So we have $F_{0}: D_{r^{\prime}} \times D^{n-1} \rightarrow M_{0}$. Since $F_{0}$ is an embedding we can choose a strongly pseudo-convex domain with $C^{\infty}$ boundary, $\Omega_{0}$, so that

$$
F_{0}\left(D_{r^{\prime}} \times D^{n-1}\right) \supset \Omega_{0} \supset F_{0}\left(D_{r} \times D^{n-1}\right) .
$$

On $\Omega_{0}$ we consider the holomorphic vector field

$$
L_{0}=F_{0^{*}}\left(\partial / \partial z^{1}\right)
$$

Clearly $L_{0}$ is an extension of $f_{0^{*}}(d / d z)$ from $f_{0}\left(D_{r}\right)$ to $\Omega_{0}$. By Proposition 1.1 and the remarks following it $\exists$ a vector field $L_{t}$ holomorphic with respect to the $t$-structure for small $t$, so that

$$
L_{t}\left(f_{0}(0)\right)=P_{t}\left(d f_{0} /\left.d z\right|_{z=0}\right),\left.\quad L_{t}\right|_{t=0}=L_{0}, \quad\left\|L_{t}-L_{0}\right\|_{s} \leqslant C\|\varphi(t)\|_{s} .
$$

We now consider the system of ordinary differential equations on $D$

$$
f_{t^{*}}(d / d z)=L_{t}\left(f_{t}(z)\right), \quad f_{t}(0)=f_{0}(0) .
$$

Since $L_{t}$ is close to $L_{0}$ by standard theory of ordinary differential equations [2] a holomorphic solution of this system exists if $t$ is sufficiently small.

If $f$ is not an embedding consider the graph map 


$$
g_{f_{0}}: D \rightarrow D \times M_{0}, \quad z \rightarrow\left(z, f_{0}(z)\right) .
$$

Then $g_{f_{0}}$ is an embedding and we apply what we have just done to $g_{f_{0}}$ and the deformation, $D \times M_{t}$ of $D \times M_{0}$ which is 0 in the first factor and the given deformation in the second, to get a map $\left(g_{f}\right)_{t}: D_{r} \rightarrow D \times M_{t}$.

To complete the proof we just project $\left(g_{f}\right)_{t}$ onto $M_{t}$.

We now turn to Theorem 2. In order to prove this theorem we need to examine Royden's extension of a map $f$ and see how it can be chosen to depend continuously on $f \in H(D, M)$. What we do is state, without proof, Royden's main lemma and then use this to discuss the continuity of the extension.

Lemma 2.2 [7]. Suppose $f: D \rightarrow M$ is an embedding and $0<r<1$. Then $\exists S_{f}^{r} \subset M$ a Stein manifold open in $M$, so that $f\left(\bar{D}_{r}\right) \subset S_{f}^{r}$.

Lemma 2.3. Let $f: D \rightarrow S$ be an embedding into a Stein manifold $S$. Then $\exists F: D \times D^{n-1} \rightarrow S$ an embedding so that $\left.F\right|_{D \times\{0\}}=f$ and $F$ can be chosen to depend continuously on $f$.

Proof. Embed $S$ as a closed submanifold of $\mathbf{C}^{N}$ for some $N . \varphi: S \rightarrow \mathrm{C}^{N}$. Consider $D \stackrel{f}{\rightarrow} S \stackrel{\varphi}{\longrightarrow} \mathbf{C}^{N}$. We will explicitly construct an extension for $\varphi \circ f$. Now $\varphi \circ f=\left(f_{1}(z), \ldots, f_{N}(z)\right)$ and let $D \times D^{n-1}=\left(z, w^{1}, \ldots, w^{n-1}\right)$. The map

$$
\tilde{F}\left(z, w^{1}, \ldots, w^{n-1}\right)=\left(f_{1}, \ldots, f_{N}\right)+\sum_{i=1}^{n-1}\left(0, \ldots,-f_{N}^{\prime}, \ldots, f_{i}^{\prime}\right) w^{i}
$$

is the required map. Here $-f_{N}^{\prime}$ is inserted in the $i$ th component. $\tilde{F}$ depends continuously on $f$ and is an embedding of $D \times\left\{\left|w^{i}\right|<\varepsilon_{i}\right\}$. Now for $\left|w^{i}\right|$ sufficiently small, $F\left(D \times D^{n-1}\right)$ can be made to lie in an arbitrarily prescribed neighborhood of $\varphi(S)$ in $\mathbf{C}^{N}$.

By the holomorphic retraction theorem [5] we can retract this neighborhood onto $S$ by a map $\rho$. We take $F=\rho \circ \tilde{F}$ and since $\rho$ has maximal rank we can solve $w^{i}=w^{i}\left(z, w^{j}\right), j \geqslant n$, by the implicit function theorem. Now we just make a change of scale in the $w$-variable to complete the proof.

Lemma 2.4. Let $0<r<1$ and let $M_{0}$ be compact hyperbolic. Then $\exists k$ $<\infty$ and $S_{i}^{r}, i=1, \ldots, k, S_{i}^{r} \subset D \times M_{0}$, Stein submanifolds open in $D \times M_{0}$ such that for every $f \in H\left(D, M_{0}\right)$ its graph restricted to $D_{r}$ lies in some $S_{i}^{r}$.

Proof. The space $G\left(H\left(D, M_{0}\right)\right) \subset H\left(D, D \times M_{0}\right)$ of graphs of elements in $H\left(D, M_{0}\right)$ is naturally homeomorphic to $H(D, M)$ and, hence, is compact. By Lemma $\left.2.2 \mathrm{~g}\right|_{D_{r}} \subset S_{g}^{r}$. We take an open set in $G\left(H\left(D, M_{0}\right)\right), U_{g}$, consisting of all mappings whose restrictions to $\bar{D}_{r}$ have graphs lying in $S_{g}^{r}$. Then $\left\{U_{g}\right\}_{g \in G\left(H\left(D, M_{0}\right)\right)}$ form an open cover. We get the result by taking a finite subcover.

Proof of TheOREM 2. Without loss of generality we may assume the deformation space is $0 \leqslant t \leqslant 1$. Fix $1>r^{\prime}>r$ and apply Lemma 2.4 to get $S_{i}^{r^{\prime}}, 1, \ldots, k$. 
We define

$$
\begin{aligned}
t_{i}^{r}\left(f_{0}\right)=\sup \left\{0<t \leqslant 1: f \text { can be deformed to } f_{t}: D_{r} \rightarrow M\right. \\
\\
\text { if we take the extension } \left.F_{0} \text {, of } g_{f_{0}}, \text { into } S_{i}^{r^{\prime}}\right\} .
\end{aligned}
$$

It is clear from Lemma 2.3 and the method of proof of Theorem 1 that if we consider our mappings being extended into a fixed $S_{i}^{r^{\prime}}$ then $t_{i}^{r}\left(f_{0}\right)$ is a continuous function on those mappings whose graphs lie in $S_{i}^{r^{\prime}}$. Call this class of mappings $\delta_{i}^{r^{\prime}}$.

We define the function $t^{r}: H\left(D, M_{0}\right) \rightarrow(0,1]$ by

$$
t^{r}\left(f_{0}\right)=\min _{i} t_{i}^{r}\left(f_{0}\right), \quad f \in \mathcal{S}_{i}^{r^{\prime}}
$$

$t^{r}$ is not continuous on $H\left(D, M_{0}\right)$ for suppose we have a sequence $f_{j}$ $\in \cap_{i=1}^{k} \delta_{i}^{r^{\prime}}$ and in $H\left(D, M_{0}\right), f_{j} \rightarrow f \in \cap_{i=1}^{k+1} \delta_{i}^{r^{\prime}}$. Then possibly $t^{r}(f)$ $<\lim _{j} t^{r}\left(f_{j}\right)$.

However this semicontinuity is sufficient to conclude that $t^{r}\left(f_{0}\right) \geqslant C>0$, for suppose $t^{r}\left(f_{0_{j}}\right) \rightarrow 0$ for some sequence $f_{0_{j}} \in H\left(D, M_{0}\right)$. By compactness we may assume $f_{0_{j}} \rightarrow f$. But then $t^{r}(f)=0$, a contradiction to Theorem 1 .

Proof of Theorem 3. Since $M_{0}$ is compact hyperbolic, $F_{M_{0}}$ is continuous on $\delta M_{0}$ [6]. The sphere bundle being compact we get $0<C_{1}<F_{M_{0}}(x, \xi)<C_{2}$ $<\infty$. Let $(x, \xi) \in \delta M_{0}$.

By Remark (b) in $\S 1$ let

$$
f_{0}: D_{F_{M_{0}}(x, \xi)^{-1}} \rightarrow M_{0}
$$

be so that $f_{0}(0)=x, f_{0}^{\prime}(0)=\xi$.

We now deform $f_{0}$. We can, by Theorem 2, take $t$ sufficiently small so that all $f_{0}: D \rightarrow M_{0}$ can be deformed to $f_{t}: D_{1-\varepsilon k} \rightarrow M_{t}$ where $k$ is to be determined. Then by change of scale all $f_{0}: D_{R} \rightarrow M_{0}$ can be deformed to $f_{t}: D_{R-\varepsilon k R} \rightarrow M_{t}$.

To prove the result we need $1 /(R-\varepsilon k R)<1 / R+\varepsilon$ or $k<R /(\varepsilon R+1)$. So take $k=\left(1 / C_{2}\right)\left(C_{1} /\left(C_{1}+\varepsilon\right)\right)$ and we are done.

\section{BIBLIOGRAPHY}

1. R. Brody, Thesis, Harvard Univ., Cambridge, Mass., June, 1975.

2. E. A. Coddington and N. Levinson, Theory of ordinary differential equations, McGraw-Hill, New York, 1955. MR 16, 1022.

3. G. Folland and J. J. Kohn, The Neumann problem for the Cauchy-Riemann complex, Princeton Univ. Press, Princeton, N.J., 1972.

4. P. A. Griffiths, Differential geometry and complex analysis, Proc. Sympos. Pure Math., vol. 27, part 2, Amer. Math. Soc., Providence, R.I., 1975, pp. 43-64.

5. R. Gunning and H. Rossi, Analytic functions of several complex variables, Prentice-Hall, Englewood Cliffs, N.J., 1965. MR 31 \#4927.

6. H. L. Royden, Remarks on the Kobayashi metric, Several Complex Variables, II (Proc. 
Internat. Conf., Univ. of Maryland, College Park, 1970), Lecture Notes in Math., vol. 185, Springer, Berlin, 1971, pp. 125-137. MR 46 \#3826.

7. The extension of regular holomorphic maps, Proc. Amer. Math. Soc. 43 (1974), 306-310. MR 49 \#629.

8. C. Seabury, Some extension theorems for regular maps of Stein manifolds, Bull. Amer. Math. Soc. 80 (1974), 1223-1224. MR 50 \#2565.

9. M. Wright, The Kobayashi pseudo-metric on algebraic manifolds of general type and in deformations of complex manifolds, Trans. Amer. Math. Soc. (to appear)

Department of Mathematics, University of Utah, Salt lake City, Utah 84112 